\title{
Efficient Cost Optimization Algorithm InIaas Cloud by Load Balancing
}

\author{
Kanaga Suba Raja $S^{a}$, Valarmathi $K^{b}$, Sritharni $C^{c}$, and Shanmugapriya $\mathbf{R}^{\mathrm{d}}$ \\ A, b,c,d \\ Department of Information Technology,Easwari Engineering College,Chennai, \\ India
}

Article History: Received: 11 January 2021; Accepted: 27 February 2021; Published online: 5 April 2021

\begin{abstract}
The distributed computing innovation is quickly growing these days because of its adaptable highlights of programmed arranging, uninhibitedly extending, on-request asset designating, force and value sparing, which just hits the objective of different associations on IT framework development. In the interim, virtualization innovation has firm relationship with distributed computing as a result of the regular idea of virtualization highlight. Virtualization innovation is much of the time used to progressively deal with load adjusting of the cloud framework where it gets conceivable to remap virtual machines (VMs) and physical assets as indicated by the adjustment in load. Nonetheless, in order to understand the least difficult presentation, the virtual machines need to completely use its administrations and assets by adjusting to the distributed computing condition powerfully. The heap adjusting and legitimate portion of assets must be ensured in order to upgrade asset utility. Numerous analysts in the past have proposed distinctive booking and cost enhancing calculations like static, dynamic and blended methodologies like best fit diminishing, first fit diminishing, however they don't ensure ideal arrangements. This task gives a cost decrease system and empowers successful burden balance. The technique utilized in this paper will propel the framework.
\end{abstract}

\section{Introduction}

Circulated registering may be an advancement that passes on figuring as organization, as opposed to a thing, where shared resources, information and writing computer programs are given over the framework. The cloud may be a pool of heterogeneous resources [1]. Its providers pass on the applications over the web, which are open from any program related with web the standard of the organization gave has improved since the heaviness of managing the advantages and their executions has been moved to the provider of the organization. Along these lines appropriated registering model has been of an immense bit of leeway to the top customers, IT buyers, programming architects, structure directors and other corporate clients since it incorporates low operational cost, ensures availability of pool of advantages, freed from cost of capital and security. Circulated figuring resources are provisioned to the top customers in pay-per-see premise.

The expert associations have and manage the data Centers at various regions, and these worker homesteads could in like manner be masterminded with different gear depending on its use. The gear similarly keeps changing with time dependent upon the customer essential. Cloud load changing implies scattering client requests over various application laborers that are running during a cloud space [2]. Cloud pro communities keep up the organization level comprehension by arranging the available resources beneficially and along these lines the time execution improvement is cultivated by sending the contraption on real virtual machines dependable with the organization level comprehension. Compelling allocation of virtual machines is in two separate advances: (a) static masterminding from the outset: bundle the course of action of virtual machines; portray them, and sending on physical host. (b) provisioning of benefits effectively: dependent upon the rest of the job needing to be done; virtual machines are made and extra resources are administered continuously.

Cloud load are every now and again balanced by serving the requesting got to the closest worker ranch. This philosophy is named Closest Data Center procedure. It diminishes the framework costs and has serving ability to a limited locale of sales age.

It is possible to remap virtual machines (VMs) and physical resources consistent with the alteration in load by using virtualization development in load changing the entire cloud system. In any case, to comprehend the least troublesome introduction, the virtual machines need to totally utilize its organizations and resources by changing in accordance with the conveyed figuring condition capably. The stack altering and genuine dissemination of advantages must be guaranteed so as to improve resource utility

At whatever point a replacement task goes into the cloud, it'll be inside the endeavor line from the start then virtual machine chief arrangements with all the new data. It'll circulate all the new data to perfect virtual machine. Each virtual machine will have least edge and most outrageous edge. If a virtual machine has achieved its most extraordinary breaking point, by then it can't get any increasingly drawn out new data. Along these lines we will guarantee flooding of data.

\section{Related Work}

As a significant part of distributed computing research, the capacity has pulled in wide consideration. What's more, a great deal of investigates have been finished by numerous researchers and foundations. 
There are a couple of issues in utilizing single cloud supplier, they are accessibility of administrations, information [7].Better issues, may utilize numeroussuppliers that gives processing, industrious capacity, and system administrations with various boundaries, for example, cost and execution [8].

Being spurred by these different boundaries, programmed determination of virtual machines inside various server farms which gives its stockpiling capacity and client necessities are proposed to decide costs versus idleness and cost versus performance.[9]

Various going before examines endeavored to viably exploit utilizing different CSPs to store information across them. RACS [10] used deletion code to limit relocation cost if either financial disappointment, blackouts, or CSP exchanging occurs. Hadji [11] thought of a couple of copy position calculations to expand accessibility and versatility for encoded information pieces while upgrading the capacity and correspondence cost. None of these frameworks investigate limiting expense by abusing estimating contrasts across various cloud suppliers with a few stockpiling.

We are here explain the inspiration driving accomplishing this work through examination of the best in class considers concentrated on the worth improvement of information in cloud related information sources.

First Come First Serve system [5] utilized stockpiling administrations during a solitary information store. The system didn't use estimating contrasts across information stores offered a few stockpiling classes. The appropriate response conveyed in FCFS isn't relevant for our cost enhancement issue. This is regularly in light of the fact that (I) FCFS needn't to influence inertness limitation, potential movement cost, and upgrading composing cost inside the instance of inevitable change.

Length store[6] improved esteeming contrasts foreordained idleness device is assured. Alimit things not reference make requests, these lines didn't require.In like manner used estimations depending on remarkable weight desire. Not exactly equivalent to virtual machines data laborers stores of expense reinforced the articles remaining main job. This causes object developments between virtual machines asserted inside the identical/different data laborer during a lone cloud. Moreover, our two estimations acknowledge a limited/no data on objects remaining job needing to be done.

Cosplay [12] propelledthe board having spot with a lone exchangingoccupations (Expert)impersonationsassertedcustomers. Like didn't utilize movement limit.[13]As opposed to our work, their answer kept an eye on simply examine simply remarkable jobs that needs to be done.

Different online estimations have been concentrated to understand different issues, for instance, dynamic provisioning in DCs [14], imperativeness careful extraordinary specialist provisioning [15] and weight changing[16]. Figuringsgottenstructure choose when a specialist executedessentialness usage, while we revolve around expense smoothing out of cloud which goes with various contributing components.

Theproblemsentinvestigations isn't appropriate in our model since it settles on a choice on schedule (for instance., when a worker is killed or when information are moved from capacity to store), while we'd prefer to shape a two-crease choice (time and spot) to work out when information ought to be moved and to which DC(s). To settle on this choice, we put forward a calculation that enhance cost. We likewise structured another calculation dependent on voracious technique[15], [17] to lead dynamic relocation of articles in this manner diminishes cost utilization.

In [17], the creators proposed on the web and disconnected calculations to upgrade the steering and situation of enormous information into the cloud for a MapReduce-like handling, with the goal that the expense of preparing, stockpiling, transmission capacity, and postponement is limited. They additionally considered movement cost of information dependent on required chronicled information that ought to be prepared along with new information created by a worldwide galactic telescope application. Rather, our work centers around enhancing the heap balance between virtual machines. Outstanding burdens ( as far as peruses and composes) on various articles.

Paper center around compromises between various assets cost. The first is figure versus capacity compromise that decides when information ought to be put away. The subsequent compromise is reserve versus capacity as sent in MetaStorage [4] that made a harmony somewhere in the range of inertness and consistency. This examination has an alternate objective, and moreover it didn't propose an answer for the cases where the remaining task at hand is obscure. The third compromise can be data transfer capacity versus store as by one way or another reenacted in DeepDive [18] that productively and rapidly recognizes target.

Virtual machine can be relocated starting with then onto the next so as to give adaptation to non-critical failure, load adjusting, framework versatility, and vitality sparing. VM relocation can be either live or non-live. The current movement approach gives around zero vacation for administration given to the applications during relocation, though the last one string and enactment of uses before moving a memory picture to the goal have. Intrigued perusers are alluded to overview papers [19], [20] for itemized conversation on VM relocation methods.

Correspondingly, information movement is arranged into two methodologies. The principal approach is live information relocation. This methodology permits that while information relocation information available clients for peruses.Information movement requests exact coordination when clients perform peruse and compose activities during the relocation procedure still it limits execution degradation[21]. As of late, live information relocation approaches are misused for value-based databases inside the setting of cloud [22], [23].

The subsequent methodology is information movement.Methodology evaluated reproduction and relocation procedures [21]. The two methods, information movement,information available clients peruses. Be that as it may, these procedures contrast in their capacity to deal with composes. In the previous, the composes are quit 
during information movement, while in the last the composes are served through a log which brings about a fiscal expense.

\section{System Model and Problem Definition}

We quickly talk about goals of the framework, and afterward dependent on which we figure an information stockpiling

\subsection{Challenges and Objectives}

Information Design is the framework wherein a client organized depiction of the information changes over. This plan is fundamental to maintain a strategic distance from bungles in information input framework and show right bearing to ace fitting data electronic structure.

The objective of organizing input is to shape information territory less troublesome and to be liberated from goofs. The information segment screen is masterminded with the objective that all the information oversee can be performed. It moreover gives record seeing working environments.

Precisely when the information enters it will check for its realness. Through screens pieces of information are entered. Fitting messages areprovided,when required with the target that the client won't be in convolution. As such the target of information course of action is to make a taking in structure that is certainly not difficult to follow.

\subsection{Input and Output:}

The information game plan is that the relationship between the information framework and consequently the client. Decisions and procedures are made for information accessibility and individuals steps are basic to put exchange information into a usable structure to process. This managing are routinely polished by reviewing the PC to analyze information from a made, printed record or it can happen by having individuals entering the information truly into framework. The course of action of information spins around controlling the extent of information required, supervising messes up, keeping up an imperative decent ways from delay, diverting additional techniques and it keeps the system clear. Information is orchestrated so it outfits security and convenience with utilizing its affirmation. Information Design thought about the accompanying things:

Information info?

List a few stages to follow when mistake happens?

What are the techniques for getting ready approval list?

Convincing and sharp thing arrangement improves the framework's relationship to help client dynamic.

1.Planning

2.Choosing Procedure.

3.Different affiliations must be made that contains data passed on .

Thing information structure should achieve in any event one targets.

Provides data conjectures.

Critical, openings, alarms.

Prompt a movement.

Confirm a movement.

\section{Implementation.}

\section{STRATEGY:}

A virtual machine has minimum threshold and maximum threshold, If a VM is having tasks in between minimum and maximum threshold then it is said to be a optimal VM.

In the event that the host load has been high to such an extent that the framework execution will decrease. We embrace the accompanying system as the accompanying

Step1: If the VM has arrived at its greatest limit, right off the bat we lock the VM and keep it from getting the new errands.

Step 2: Check nearby VM is in optimal state .

Step 3: To avoid overflow of tasks, new tasks are migrated on new VM which is in optimal state.

If the VM is having tasks lower than minimum threshold then,

Step 1: Migrate the tasks to another VM which is in optimal stage.

Step 2: After freeing the VM, suspend the VM from usage.

MODULE:

This Project has One Module and they have two roles to play.

It is

- Load balancing

- Cost optimization

Load Balancing:

Cloud Sims is a structure for making and copying distributed computing frameworks and administrations. Initially worked at the Cloud Computing and Distributed Systems.As a common smoothing out issue with the goal of diminishing overhead figuring over the device. In this framework we have 3 workers where these three 
workers have their own ability of giving the solicitation sent by customer. In the event that solicitation goes past the limit of worker (1) at that point consequently that worker diverts it to the following worker (2) this empowers the heap adjusting of workers and taking care of solicitation of the worker in a simple way and furthermore utilization of intensity is finished by closing down the unused virtual machines. Here there will a host and under that host number of virtual machines can be found. Each virtual machines has their own limit esteems and utilizing this we can discover the maximum burden and min limit of every individual VM so if the edge esteem is higher than Virtual machine.It can actuate another VM for moving of burden this lessens the heap limit of VM(1). On the off chance that the heap limit of $\mathrm{VM}(1)$ is lower and furthermore $\mathrm{VM}(2)$ is running with min edge esteem at that point if $\operatorname{VM}(1)$ has the ability to acknowledge the solicitation from $\mathrm{VM}(2)$ so cost utilization for $\operatorname{VM}(2)$ can be spared.

Cost Optimization :

Considering high transmission capacity deferral to end ,center around the rising solicitation portion issue in geologically dispersed server farms, and propose a combine development model. In particular, we present NBS based technique for both the prerequisite of supplier's high transfer speed use at all server farms and end-clients' low postponement.

We propose a productive solicitation designation calculation by acquainting the helper variable strategy with dispense with imbalance, as opposed to by legitimately applying the Logarithmic Smoothing procedure. We perform hypothetical investigation to demonstrate the nearness and uniqueness of our answer, and besides association of our computation.

By utilizing our solicitation allotment strategy we apportion undertakings to virtual machines that are ideal and the unused virtual machines are suspended, in this manner cost can be diminished somewhat.

We lead a lot of tests dependent on genuine remaining task at hand follows. With the reproduction results, we show that our calculation surpasses the ordinary insatiable and region calculations, and can proficiently improve the move speed use customers.

\section{CloudSim Simulation Toolkit:}

Distributed computing might be a compensation as you utilize model, which conveys foundation, stage and programming as administrations to clients according to their necessities. Distributed computing uncovered server farms abilities as system virtual administrations, which can incorporate the arrangement of required equipment, application with help of the database likewise on the grounds that the interface . this empowers the clients to send and get to applications over the web which is predicated on request and prerequisites.

\subsection{Why use Cloudsim?}

A few test systems are frequently wont to recreate the working of most recent administrations, among them. CloudSim Simulation Toolkit is that the more summed up and compelling test system for testing Cloud figuring related theory. An extensible recreation structure created by a group of specialists at CLOUDSLaboratory, Melbourne. This toolbox permits consistent demonstrating, recreation, and Experimentation related with cloudbased foundations and application administrations and its different discharged variants are distributed on Cloudsim's GitHub venture page.

This reproduction toolbox permits the analysts additionally as cloud designers to check the presentation of the potential cloud application for execution testing during a controlled and clear to arrangement condition. And Furthermore permits calibrating the overall help execution even before it's sent inside the creation condition.

Features of CloudSim Simulation Toolkit:

Backing recreation huge scope conditions,serverfarms, one physical registering node(could be a work area, PC, or worker machine).

2. It is a stage for displaying Clouds, administration representatives, provisioning, and portion approaches.

3. Encourages the recreation.

4. Reenactment of united Cloud condition is encouraged.

5. Accessibility of a virtualization motor that encourages the creation and the board of various, free, and cofacilitated virtualized administrations on an information community hub.

6. Adaptability to change between space-shared and time-shared allotment of handling centers to virtualized administrations

\section{PROPOSED SYSTEM:}

We invest critical energy in the creating sales partition issue in topographically circled improvement deferral clients. In particular, we present Nash exchanging strategy (NBS) based methodology to show both the need'; of supplier's high transfer speed use and clients deferral.

$\square$ Figure requesting assignment necessities improvement issue. advancement are frequently NP-hard.we propose an effective solicitation allotment calculation by acquainting the assistant variable strategy with dispense with imbalance.

$\square$ Lead an outsized measure of tests upheld true outstanding task at hand follows. By having our reenactment results, Our calculation plays out the covetous and area calculations, and improve the transfer speed usage for supplier and diminish the deferral for end-clients productively.

ADVANTAGE:

1. Shifting remaining burden from over-burden datacenters with low use 
2. Their Delay doesn't contain the reaction time inside server farms

FLOW CHART DIAGRAM:

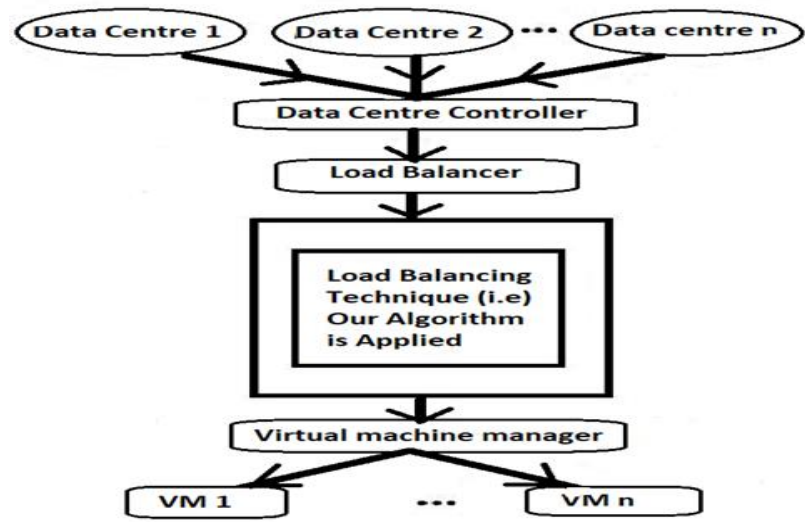

ARCHITECTURE DIAGRAM

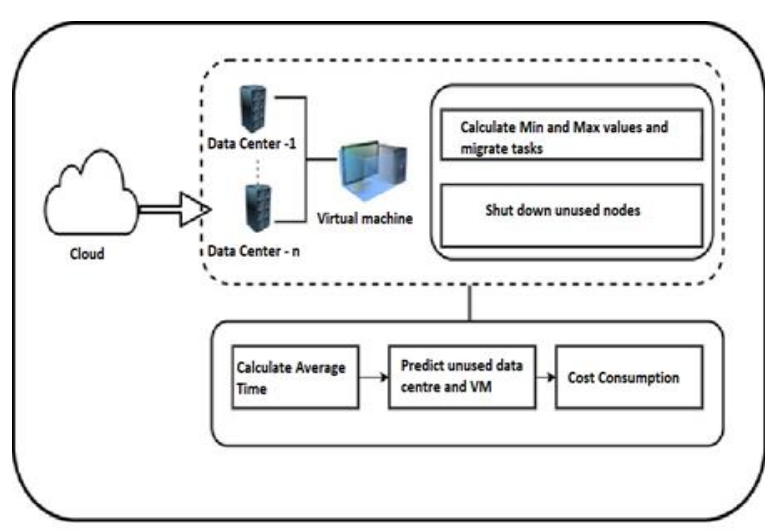

\section{ALGORITHMS:}

\subsection{Algorithm for cost utilization:}

Information:

A text $R=\{R 1, R 2$ $, \mathrm{Rn}\}$, array s.

Yield:

Discover term outpace, to foresee VMTHEFT

Centers $\{01, \mathrm{O} 2$, .,On $\}$ essentially segregateR into s arrays.

Calculation:

Boot the text

Break the text into 's'divisions

$\mathrm{s} \square$ is positive integer

Choose s initial centers $\mathrm{O}=\{\mathrm{O} 1$, On $\}$

While halting benchmark has not been met

Do $\square$ task step:

For $\mathrm{i}=1, \ldots \ldots \ldots, \mathrm{N}$

Do discover storage room focus osOto occurrence ri

Appoint example ri to set Os.

End for

For $\mathrm{i}=1, \ldots \ldots \ldots, \mathrm{s}$

Accomplish designationworking time

Normal Time VMi $=$ Tmin $-\mathrm{Tmax}$ 
Cost $\mathrm{xi}=$ Tavg

Cost $\mathrm{yi}=$ Tavg

Halt

AnticipateVMTHEFTdependent leastterm surpasses.

Bring groups back.

\subsection{Algorithm for vitality utilization:}

Information:

Normal season of Virtual Machine.

Yield:

To figure the vitality utilization of Virtual Machines.

Calculation:

Introduce $\mathrm{j}$

While

Do $\square$ load work:

For $\mathrm{i}=1, \ldots \ldots \ldots, \mathrm{N}$

Discover storeroom working time

$\mathrm{Ei}=$ Start time - End Time.

Vitality $=\mathrm{W} * \mathrm{~S}$;

$\mathrm{W} \square$ Amount of work Done and $\mathrm{S} \square$ Slab Capacity

Halt

Calculate $\square$ Energy Consumption of virtual machine.

End.

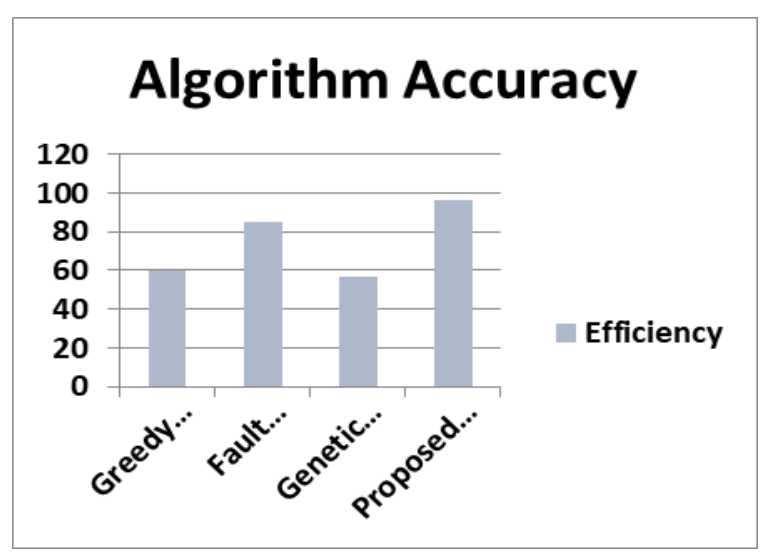

\section{Graphs and Results.}

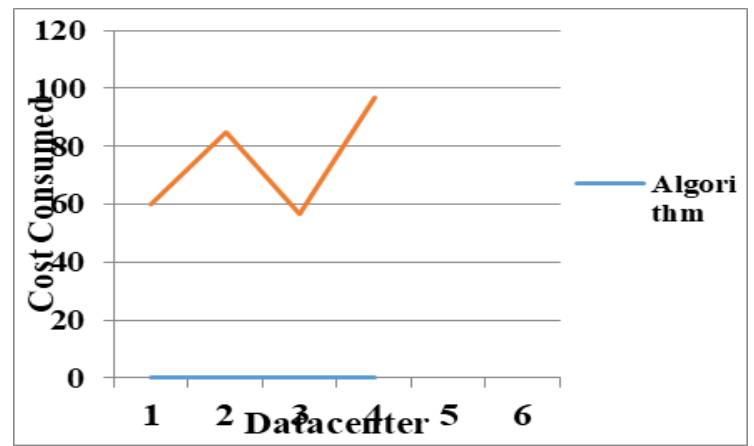

Above graph represents cost optimization on the vertical axis and data centers on the horizontal axis. Above graph represents the efficiency of the proposed algorithm. X axis consist of virtual machines and $\mathrm{Y}$ axis consist of simulation time. Efficiency of simulation time is represented here. 


\section{Conclusions and Future Work}

Our focus right now the difficult assignment in topographically dispersed datacenters.Specifically, the provider's need of high exchange speed utilization at all datacenters and clients low delay conditions are both shown subject to the Nash wheeling and dealing game. By then, we characterize the structure of testing task under those conditions as a headway issue, which is an entire number upgrade similarly as NP-hard. To capably deal with such a smoothing out issue, we propose a sales divide computation by acclimating partner factors with take outimbalancing approval, instead of precisely applying the Logarithmic Smoothing strategy. Speculative examination exhibits the nearness and uniqueness of our optimal game plan and the entwining of our estimation. We precisely decide computation reliant on genuine exceptional weight follows. The starter results show that our computation can capably improve the information move limit use for provider additionally delay for customers can be made diminish, differentiated both energetic and region calculations. As future work, we intend to improve all the more altogether and study the decentralized execution of solicitation designation by conveying one's controller in each datacenter.

\section{Reference:}

1. S. Muralidhar, et.al., "f4: Facebook's warm blob storage system," in Proc. 11th USENIX Symp. Operating Syst. Des. Implementation., Oct. 2014, pp. 383-398.

2. Bourdena,et.al.,"Using socio-spatial context in mobile cloud offload process for energy conservation in wireless devices,” IEEE Trans. Cloud Comput., 2016, Doi: 10.1109/TCC.2015.2511736.

a. Kathpal,et.al.,"Analyzing compute versus storage tradeoff for video-aware storage efficiency," in Proc. 4th USENIX Conf. Hot Topics Storage File Syst., 2012, pp. 13-13.

3. D. Bermbach, et.al., "MetaStorage: A federated cloud storage system to manage consistency-latency tradeoffs," in Proc. IEEE 4th Int. Conf. Cloud Comput., 2011 , pp. 452-459.

4. K. P. Puttaswamy, et.al., "Frugal storage for cloud file systems," in Proc. 7th ACM Eur. Conf. Comput. Syst., 2012, pp. 71-84.

5. Z. Wu, et.al., and H. V. Madhyastha, "SPANStore: Cost-effective geo-replicated storage spanning multiple cloud services," in Proc. 24th ACM Symp. Operating Syst. Principles, 2013, pp. 292-308.

6. N. Toosi, et.al., "Interconnected cloud computing environments: Challenges, taxonomy, and survey," ACM Comput. Surveys, vol. 47, no. 1, pp. 7:1-7:47, May 2014.

a. Li, et.al., "CloudCmp: Comparing public cloud providers," in Proc. 10th ACM SIGCOMM Conf. Internet Meas., 2010, pp. 1-14.

b. Ruiz-Alvarez, et.al., "A model and decision procedure for data storage in cloud computing," in Proc. 12th IEEE/ ACM Int. Symp. Cluster Cloud Grid Comput., 2012, pp. 572-579.

7. H. Abu-Libdeh, et.al., "RACS: A case for cloud storage diversity," in Proc. 1st ACM Symp. Cloud Comput., 2010, pp. 229-240.

8. M.Hadji,et.al.,ScalableandCost Efficient AlgorithmsforReliableandDistributed Cloud Storage. Cham, Switzerland: Springer, 2016, pp. 15-37.

9. L. Jiao, et.al, "Optimizing cost for online social networks on geo-distributed clouds," IEEE/ACM Trans. Netw., vol. 24, no. 1, pp. 99-112, Feb. 2016.

10. F. Chen,et.al.,"Intra-cloud lightning: Building CDNs in the cloud," in Proc. IEEE INFOCOM, Mar. 2012, pp. 433-441.

11. T. Lu, et.al., "Simple and effective dynamic provisioning for power-proportional data centers," IEEE Trans. Parallel Distrib. Syst., vol. 24, no. 6, pp. 1161-1171, Jun. 2013.

12. M. Lin,et.al., "Online algorithms for geographical load balancing," in Proc. Int. Green Comput. Conf., Jun. 2012, pp. 1-10.

13. M. Lin, et.al., "Dynamic right-sizing for power-proportional data centers," IEEE/ACM Trans. Netw., vol. 21, no. 5, pp. 1378-1391, Oct. 2013.

14. L. Zhang, et.al., "Moving big data to the cloud: An online cost-minimizing approach," IEEE J. Sel. Areas. Commun., vol. 31, no. 12, pp. 2710-2721, Dec. 2013.

15. D. Novakovic, et.al., "DeepDive: Transparently identifying and managing performance interference in virtualized environments," EPFL, Lausanne, Switzerland, Tech. Rep. 183449, 2013.

16. V. Medinaet.al.,"A survey of migration mechanisms of virtual machines,” ACM Comput. Surveys, vol. 46, no. 3, Jan. 2014, Art. no. 30.

17. R. W. Ahmad, et.al., "A survey on virtual machine migration and server consolidation frameworks for cloud data centers," J. Netw. Comput. Appl., vol. 52, pp. 11-25, 2015.

18. N. Tran, et.al., "Online migration for geo-distributed storage systems," in Proc. USENIX Conf. USENIX Annu. Tech. Conf., 2011, pp. 15-15.

19. J. Elmore, et.al., "Zephyr: Live migration in shared nothing databases for elastic cloud platforms," in Proc. ACM SIGMOD Int. Conf. Manage. Data, 2011, pp. 301-312. 
20. S. Das, et.al., "Albatross: Lightweight elasticity in shared storage databases for the cloud using live data migration," Proc. VLDB Endowment, vol. 4, no. 8, pp. 494-505, May 2011.

21. S. Das, et.al., "ElasTraS: An elastic transactional data store in the cloud," in Proc. Conf. Hot Topics Cloud Comput., 2009, Art. no. 7.

22. Sampathkumar, A., Murugan, S., Elngar, A.A., Garg, L., Kanmani, R. and Malar, A.C.J., 2020. A Novel Scheme for an IoT-Based Weather Monitoring System Using a Wireless Sensor Network. In Integration of WSN and IoT for Smart Cities (pp. 181-191). Springer, Cham 